\title{
ANALYSIS OF SPIRAL AGGREGATE DEVICE ON THE SUMP CLEANING MACHINE BY DISCRETE ELEMENT METHOD
}

\author{
Gao, K. D. ${ }^{*, * * * * *} ;$ Xu, W. B. ; Zhang, X.,"\# \& Wang, G. ${ }^{* * * *}$ \\ *College of Mechanical \& Electrical Engineering, Shandong University of Science \& Technology, \\ Qingdao 266500, China \\ ** Jiangsu Key Laboratory of Mine Mechanical and Electrical Equipment, China University of Mining \\ \& Technology, Xuzhou 221116, China \\ **** Key Laboratory of Mining Machinery Engineering of Shandong Province, Qingdao 266500, China \\ *** Department of Mining Engineering, West Virginia University, Morgantown, WV, 26505, \\ United States \\ E-Mail: zhangxin@sdust.edu.cn $\left({ }^{\#}\right.$ Corresponding author)
}

\begin{abstract}
Sump cleaning machine is important coal mine equipment designed to reduce the labour intensity of underground workers, and its front end usually uses a spiral aggregate device. However, the traditional empirical design methods cannot accurately obtain the optimal parameters and defects in design of the spiral aggregate device due to the complex structure. To improve the design approach, the discrete element numerical model of the working process of the spiral aggregate device was constructed to conduct simulation research and design three key parameters, namely, screw shaft speed, roof inclination angle of the feeding port, and number of throwing plates. Results demonstrate that the aggregate rate increases first and then decreases with the increase in the rotating speed of screw shaft. In addition, the increase in the number of throwing plates is beneficial to improve the stability of the axial movement of slime water. The roof inclination angle of the feeding port has no significant influence on the aggregate effect. The results provide guidance for improving the structure of the spiral aggregate device and a reference for optimizing the design of complex screw mechanisms.

(Received in June 2018, accepted in September 2018. This paper was with the authors 2 weeks for 1 revision.)
\end{abstract}

Key Words: Discrete Element Method, Sump Cleaning Machine, Spiral Aggregate, Conveyor

\section{INTRODUCTION}

Groundwater inrush is a coal mine safety accident that is as serious as pressure bump and gas outburst $[1,2]$. The resulting harms can be effectively alleviated by using an underground sump [3]. However, if the slime in the sump cannot be cleaned on time, it will cause serious safety risks to coal mine production. A sump cleaning machine is an important piece of equipment used to clean the sump. At present, there are various types of sump cleaning machines used in coal mines, such as pumping type, stripping type, shovelling type, and spiral aggregate type. Among them, the spiral aggregate sump cleaning machine has attracted considerable attention because of its simple structure, high efficiency, good adaptability, and strong stirring force. The spiral aggregate device is the core component of this sump cleaning machine. At present, the spiral aggregate device design is mostly based on the theory of screw conveyor. For example, $\mathrm{Hu}$ et al. have optimized the spiral aggregate device structures by using the ant colony algorithm according to the design theory of screw conveyor [4].

With the popularization of spiral aggregate sump cleaning machines, the reliability and applicability of the design method become important. However, the design of the spiral aggregate device with the traditional design theory of screw conveyor has the following defects: (1) The traditional design theory mainly aims at the closed screw conveyor, but the spiral aggregate device is half open, with different feeding modes and constraint space. (2) Slime water is a multi-phase and multi-dispersed complex system that contains the suspended 
particles, and its characteristics are between fluid and solid [5]. The existing design methods disregard the influence of material parameters. (3) The existing design methods only consider the spiral blades but not the coupling effect between the spiral blades and the throwing plates. On this basis, studying and obtaining a new method of designing and optimizing the structural of the spiral aggregate device become a key problem to be solved urgently. With the development of numerical simulation technology, the discrete element method (DEM) has been widely used in the simulation of fluid and solid materials [6-8]. Many scholars have also completed the design and performance analysis of complex mechanical structures, such as scraper conveyor [9], belt conveyor [10], feeder [11], and coal mining machine [12], by means of DEM.

In this study, a simulation model of the spiral aggregate device in collecting slime water particles was established by using DEM. The rotating speed of the screw shaft, the number of throwing plates, and the slope of roof at the aggregate port were designed and analysed to obtain the optimum design method of spiral aggregate device and the structural and working parameters of the key parts, clarify the defects in the existing methods, improve the working efficiency of the spiral aggregate device, and provide a reference for the structural optimization and improvement of the entire machine in the future.

\section{STATE OF THE ART}

Several researchers have conducted considerable studies on closed screw conveying equipment. In the aspect of theoretical calculation, Roberts [13] simplified the contact relationship among particles in a closed screw conveyor and deduced the mathematical expressions of the conveying efficiency and the screw conveyor structure by constructing movement and stress models of single particles in the screw conveyor. On the basis of the research results of Roberts, Dai and Grace [14] deduced the torque calculation expression of conveying biomaterials in a closed spiral feeder by combining with the characteristics of biomaterial objects. The theoretical research of Roberts and Dai and Grace focused on the closed screw conveying condition, in which the radial motion of particles was subject to strict spatial constraints and model simplification was easy. However, the radial randomness of half-open screw conveying particles was large, which made model simplification and theoretical derivation difficult. Lessmann and Schoeppner [15] analysed the conveying process of a single-screw extruder. They found that the friction properties and shape of materials greatly influenced the conveying performance. Nachenius et al. [16] studied the retention time of different materials in a conveyor during closed screw conveying process. Their results showed that the conveying effects of different materials varied under the same conditions. The studies of Lessmann and Schoeppner and Nachenius et al. indicated that the design of spiral aggregate device could not ignore the influence of slime water characteristics. Davison et al. [17, 18] studied the rheological characteristics of soil in a spiral drill pipe by computational fluid dynamics (CFD) method. They emphasized that the soil could be regarded as a fluid to be studied at this time. Talebi et al. [19] used CFD software to simulate the flow characteristics of sand in a screw conveyor of a shield and proved that the error between the simulated and actual torques was small through experiments. Jovanović et al. [20] studied the influence of the geometric parameters of the blades of a closed screw conveyor on the material mixing effect by using DEM and indicated that the mixing effect could be improved by adding new blades at the edge of spiral blades. Davison and Talebi et al. successfully simulated the process of screw conveying by CFD, but the simulation conditions should meet the internal pressure, filling rate, and space requirements, which were not suitable for simulating the conveying process of particles under atmospheric pressure. Although the research objects of Aca et al. presented a great difference from the spiral 
aggregate device, the results confirmed that the structure of the new spiral equipment could be optimized by DEM.

The above research results mainly focus on the theoretical calculation of parameters and the numerical simulation and optimization design of closed screw conveying equipment, but none of them involve semi-closed screw conveying equipment. The screw aggregate device is semi-closed, which is quite different from the closed screw conveyor in working mode and working principle. The existing design theories of the closed screw conveyor cannot guide the design of the spiral aggregate device, and guiding the design of the spiral aggregate device by constructing a new theoretical model is difficult. Therefore, in this study, a 3D simulation model of spiral aggregate device on a sump cleaning machine and a discrete element model of slime water particles were established by using DEM on the basis of determining the physical parameters of slime water. From the analysis of the movement behaviour of particles in the working process, the amounts and velocities of particles in the key area under different shaft speeds, the numbers of throwing plates, and the roof inclination at the feeding port were compared to find the design defects of the current model of spiral aggregate device and determine the reasonable design and working parameters, which provided basis for optimization and improvement.

The remainder of this paper is organized as follows. Section 3 establishes the simulation model of the spiral aggregate device and the discrete element model of the slime water particles, and provides the main parameters and the variable values in simulation. Section 4 presents the simulation results for the working performance of the spiral aggregate device under different design parameters, such as the rotating speed of the screw shaft, the number of throwing plates, and the roof inclination angle of the feeding port. Section 5 provides the conclusions of this study.

\section{METHODOLOGY}

\subsection{Physical model and numerical model of spiral aggregate device}

Fig. 1 presents a new type of sump cleaning machine. The machine is mainly composed of aggregate device, conveying device, hydraulic system, walking mechanism, and frame of the body. The spiral aggregate device of the sump cleaning machine is submerged in slime water to move forward, and its screw shaft rotates at the same time. It mainly relies on the axial conveying function of the screw blade to centralize the materials in the aggregate device to the throwing plate in the middle, then throws the materials into the aggregate trough with the help of the throwing plate, and finally feeds the slime water into the mine car or the filter press device on the rear side by means of the conveying device. With the help of the screw blade, the screw aggregate device can not only realize the aggregation of materials but also play the role of fully stirring and mixing slime and water.

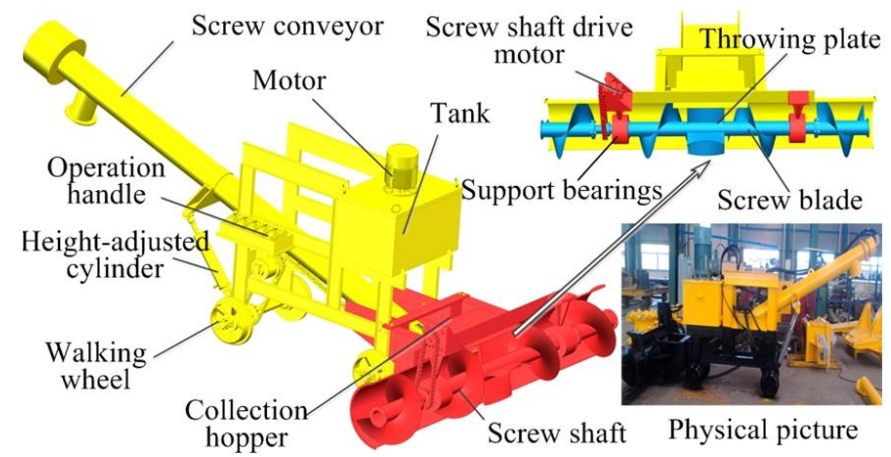

Figure 1: Sump cleaning machine model and physical picture.

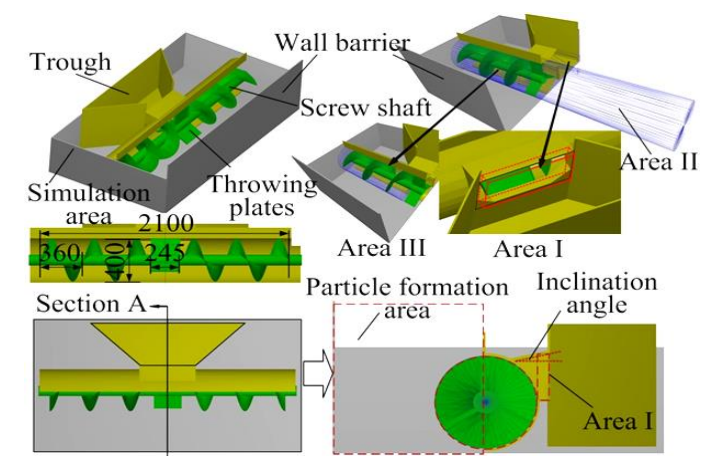

Figure 2: Simulation model of a spiral aggregate device. 
To reduce the calculation time, the screw aggregate device model was simplified on the basis of meeting the research needs, as shown in Fig. 2. The main structural features were retained in the model, but the motor, chain, and bearing were disregarded. The box-shaped simulation area and wall barrier were added to define the particle formation area. To study the influence of the rotating speed of the spiral blades, the inclination angle of the roof at the feeding port, and the number of throwing plates on the aggregate effect, three statistical regions and the inclination angle $\alpha$ of the roof at the feeding port were determined in Fig. 2. In the figure, Area I is a hexahedron, located in the channel of the feeding port. Area II is the cylindrical space enveloped by the rotational motion of the screw shaft. Area III is the cylindrical space enveloped by the rotational motion of the spiral blade on the left of the screw shaft. The feeding port in the model was designed as a channel with a closed upper roof to guide the materials into the trough. The channel has a certain slope, which meant the angle between the horizontal plane and the inner wall of the channel. The materials are blocked by the inner wall and have to turn to the channel of the feeding port in the process of oblique upward or upward movement. The position of the roof inclination angle $\alpha$ of the feeding port is shown in Fig. 2.

\subsection{DEM}

DEM and the finite and boundary element methods used for solving the complex continuous system have parallel mathematical concepts and similar physical meanings [21]. Some scholars even combined them to solve engineering problems of different scales. The basic principle is to divide the research object into elements and make each element independent of each other. The cyclic iterative computation is realized by using the dynamic or static relaxation method to determine the displacements and forces of all elements in each time unit and then update the position of each element. By tracing calculation of the micro-motion of all the elements, the whole motion law of the research object can be obtained macroscopically [22].

The granular soft sphere model was adopted in the simulation. This model does not consider the deformation of particles in contact and simplify the normal force of particles generated on the contact surface into the damping and spring and the tangential force into the slider, spring, and damping. In addition, it introduces the damping coefficient, elastic coefficient, and other physical parameters in classical physics and refers to the contact displacement of particles to calculate the contact force.

The normal contact force $F_{n i j}$ of the particle is the superposition of damping force and elastic force generated by equivalent normal spring and damping on the particle, as shown in Eq. (1):

$$
F_{n i j}=\left(-k_{n} \alpha-c_{n} v_{i j} n\right) n
$$

where, $\alpha$ is the normal overlap, $v_{i j}$ is the relative velocity of the two particles, $n$ is the unit vector that coincides with the connection between two particles $i$ and $j . c_{n}$ is the normal damping coefficient. $k_{n}$ is the normal elastic coefficient of particles, and its expression is shown in Eq. (2):

$$
k_{n}=\frac{4}{3}\left(\frac{1-v_{i}^{2}}{E_{i}}+\frac{1-v_{j}^{2}}{E_{j}}\right)^{-1}\left(\frac{R_{i}+R_{j}}{R_{i} R_{j}}\right)^{-1 / 2}
$$

where, $E$ is the elastic modulus of the material, $v$ is the Poisson's ratio of the material, $R$ is the radius of particle $i$ or $j$.

Like the normal contact force, the tangential force $F_{t i j}$ can be expressed by Eq. (3):

$$
F_{t i j}=-k_{t} \delta-c_{t} v_{c t}
$$


where, $v_{c t}$ is the slip velocity of contact points, is the tangential displacement at the contact point, $k_{t}$ is the tangential elastic coefficient, $\delta$ is the tangential displacement of the contact point, $c_{t}$ is the tangential damping coefficient.

\subsection{Contact model of particles}

The Hertz-Mindlin model combined with the JKR model was used in the simulation of the study. As a cohesive contact model, it can consider the influence of van der Waals forces in the contact area and simulate strong viscous systems, dry powders, or wet particles. In this model, the normal force component is based on Hertzian contact theory, and the tangential force model is based on Mindlin-Deresiewicz theory. Both normal and tangential forces have damping, and their damping coefficients are related to the recovery coefficient [23]. The tangential friction follows Coulomb's law of friction. The rolling friction is achieved by the contact independent directional constant torque model [24].

The calculation of the JKR normal force $F_{J K R}$ is based on the amount of overlap $\delta$ and surface energy $\gamma$, as shown in Eqs. (4) and (5):

$$
\begin{gathered}
F_{J K R}=-4 \sqrt{\pi \gamma E^{*}} \alpha^{3 / 2}+\frac{4 E^{*}}{3 R^{*}} \alpha^{3} \\
\delta=\frac{\alpha^{2}}{R^{*}}-\sqrt{4 \pi \gamma \alpha / E^{*}}
\end{gathered}
$$

where, $\delta$ is the amount of overlap, $E^{*}$ is the equivalent Young's modulus, and $R^{*}$ is the equivalent radius, $\alpha$ is the contact radius.

The expression of normal damping force $F_{n}{ }^{d}$ is shown in Eq. (6):

$$
F_{n}^{d}=-2 \sqrt{\frac{5}{6}} \beta \sqrt{S_{n} m^{*}} v_{n}^{\overline{r e l}}
$$

where, $v_{n}^{\overrightarrow{r e l}}$ is the normal component of relative velocity, $S_{n}$ is normal stiffness, $m^{*}$ is the equivalent mass,

$$
\beta=\frac{\ln e}{\sqrt{\ln ^{2} e+\pi^{2}}}
$$

where, $e$ is the recovery coefficient.

In addition, the tangential damping $F_{t}{ }^{d}$ expression is shown in Eq. (8):

$$
F_{t}^{d}=-2 \sqrt{\frac{5}{6}} \beta \sqrt{S_{t} m^{*}} v_{t}^{\overline{r e l}}
$$

where, $v_{t}^{\overrightarrow{r e l}}$ is the tangential component of relative velocity, and the tangential force is limited by the coulomb friction force, $S_{t}$ is the tangential overlap.

\subsection{Simulation parameters and simulation process}

Two attributes of materials were set in the simulation, namely, steel and slime water. They were used to define the geometric model of the spiral aggregate device and the slime water particles, respectively. The main differences between the two material parameters are in shear modulus and density, where the shear modulus of steel is $7.9 \times 10^{9} \mathrm{~Pa}$, the density is $7850 \mathrm{~kg} / \mathrm{m}^{3}$, and the slime water is set to $1.98 \times 10^{9} \mathrm{~Pa}$ and $1100 \mathrm{~kg} / \mathrm{m}^{3}$. In addition, both materials have a Poisson's ratio of 0.3 and a work function of 0 . Contact parameters between materials mainly include restitution coefficient, static friction coefficient and rolling friction coefficient. In this study, these three coefficients are set to $0.2,0.2$ and 0.01 , respectively. The 
slime particles were selected as the sphere model with a radius of $8 \mathrm{~mm}$ for simulation. The mass of a single particle was approximately $2.36 \mathrm{~g}$, and its volume was $2.14 \mathrm{~cm}^{3}$.

At the beginning of the simulation, the particles were generated in the particle formation area, and they freely dropped and stood. The trough and screw shaft were stationary. At $1.5 \mathrm{~s}$, they moved to the positive direction of $\mathrm{x}$ axis (i.e., the direction of the particles) at a speed of $0.04 \mathrm{~m} / \mathrm{s}$, which simulated the forward motion of the entire machine. When the model moved in a straight line, the screw shaft rotated around its geometric axis to simulate the real working state of the spiral aggregate device. In this study, several groups of simulation were performed with the rotating speed of the screw shaft as a variable.

Through analysis of the simulation results, the optimal operating speed of the spiral aggregate device was obtained. The total simulation time was set to $4 \mathrm{~s}$. It was the free-fall time of the particles in the first $1.5 \mathrm{~s}$. After this period, the speed of the particles decreased and became static, which accorded with the accumulation state of the real situation. The particles were then used to prepare for subsequent simulations. In the research, the simulation was divided into three parts. In the first part, the aggregate effects of the aggregate device at rotating speeds of $166,200,215,230,250$, and $266 \mathrm{rpm}$ were studied. The slope of the feeding port was $13^{\circ}$, the number of throwing plates was 3 , and the simulation time was $4 \mathrm{~s}$. In the second part, the aggregate effects of the aggregate device with two, three, four, and five throwing plates were studied. At this time, the rotating speed of the screw shaft was $230 \mathrm{rpm}$, and the slope of the feeding port was $13^{\circ}$. In the third part, the aggregate effects of the aggregate device with the slopes of the feeding port being $7^{\circ}, 9^{\circ}, 11^{\circ}$, and $13^{\circ}$ were studied. At this time, the rotating speed of the screw shaft was $230 \mathrm{rpm}$, and the number of the throwing plates was 3 .

\section{RESULT ANALYSIS AND DISCUSSION}

\subsection{Influence of the rotating speed of the screw shaft on the aggregate effect}

Fig. 3 shows the curves in which the average velocity of particles in Area I varied with time when the rotating speeds of the screw shaft were 166, 200, 215, 230, 250, and $266 \mathrm{rpm}$. According to the linear fitting results of six groups of curves in Fig. 3, the residual sum of squares $(R S S)$ increased with the rotating speed of the screw shaft, which is reflected by the wide fluctuations of the average velocity curve of particles in Area I around the regression line with the increase in the rotating speed of the screw shaft. Its physical meaning is that with the increase in the rotating speed, the velocity fluctuation of particles flowing into the channel increases gradually. Because the force acting on the throwing plate by particles does not affect the rotating speed of the screw shaft, it could be deduced from the above results that the motion of the throwing plate is faster than the axial flowing of particles, leading to the lower flowing rate of particles compared with the conveying capacity of the throwing plate. The throwing plate completes the short-time working on particles through discontinuous impact on the particle flow in axial movement, which enables the particles to obtain the speed of moving along the direction of the feeding port.

To obtain the optimal value of the rotating speed of the spiral blade, 200 time points were taken out evenly from $1.5 \mathrm{~s}$ to $4 \mathrm{~s}$ of the simulation. The number and velocity of particles in Area I were collected to draw the scatter plot and conduct linear regression analysis. The results are shown in Fig. 4. In the simulation, the size and mass of each particle are the same, and the mass can be expressed by the number of particles. Therefore, the distribution of particles in Fig. 4 could be used to judge the kinetic energy of particles. We assumed that the speed of particles is positively correlated with the number of particles. In other words, the particle flow with larger mass could pass through the feeding port at a faster speed. In the 
regression analysis (Fig.4), the Pearson correlation coefficient is used as the mathematical standard to evaluate this characteristic.

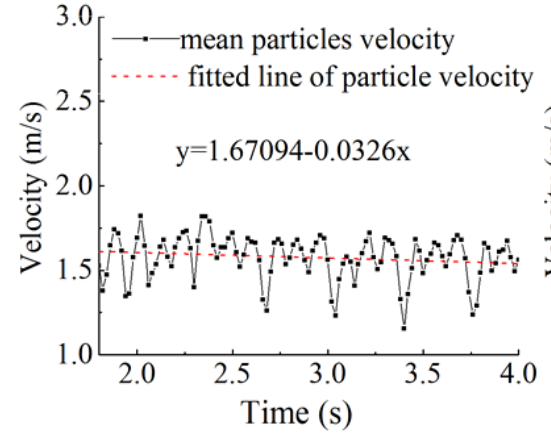

a) Rotation speed $166 \mathrm{rpm}$

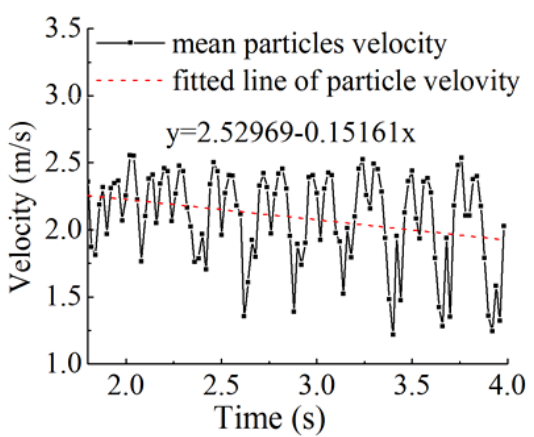

d) Rotation speed $230 \mathrm{rpm}$

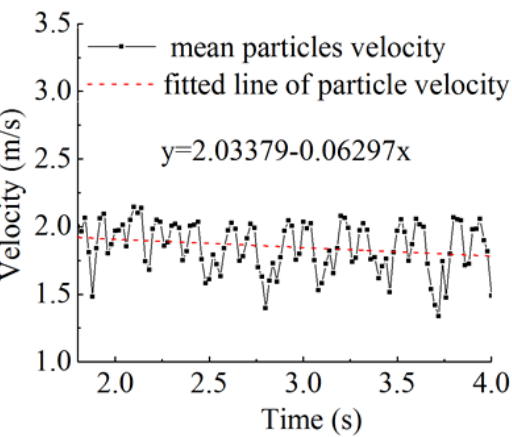

b) Rotation speed $200 \mathrm{rpm}$

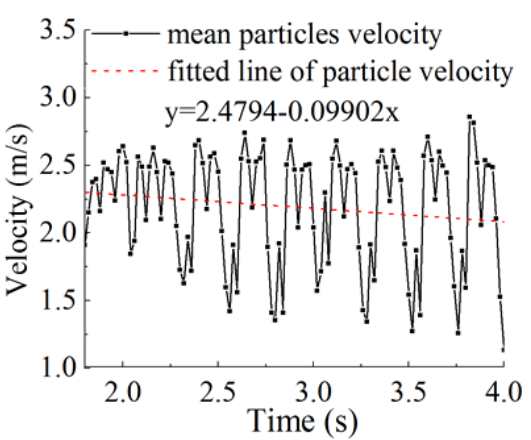

e) Rotation speed $250 \mathrm{rpm}$

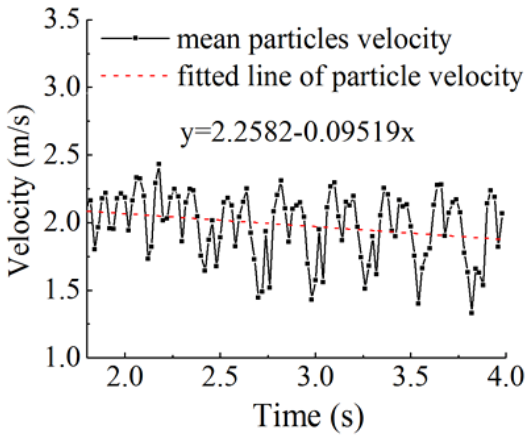

c) Rotation speed $215 \mathrm{rpm}$

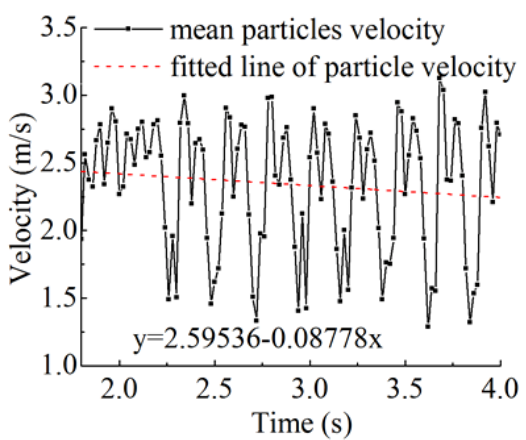

f) Rotation speed $266 \mathrm{rpm}$

Figure 3: Average velocity of particles in Area I varied with time in different rotation speed.

As shown in Fig. 4, the Pearson correlation coefficient of each group first increases and then decreases with the increase in the rotating speed of the screw shaft, and the value is the largest when the rotating speed of the spiral blade is $230 \mathrm{rpm}(0.81414)$. In addition, as the coefficient is close to the value of 0.80294 at the rotating speed of $250 \mathrm{rpm}$, we refer to the $R S S$ of the two regression calculations. The RSS is 582996 when the rotating speed of the spiral blade is $230 \mathrm{rpm}$, slightly smaller than that (686177.95) when the rotating speed is $250 \mathrm{rpm}$. This result indicates that the group of data at the rotating speed of $230 \mathrm{rpm}$ is more concentrated on the regression line, and the interpretation degree of linear regression is higher. The working of the throwing plate on particles is characterized by short time and fast speed. Accordingly, the high rotating speed of the screw shaft does not indicate a good aggregate effect of the aggregate device. This is because the change of the conveying capacity of the spiral blade with the rotating speed of the screw shaft is not completely consistent with the change in the particle transfer capacity of the throwing plate with the rotating speed of the screw shaft. Their combination ultimately determines the aggregate efficiency. The axial conveying capacity of the spiral blade is better than the particle transfer capacity of the throwing plate at a low rotating speed. However, with the increase in the rotating speed of the screw shaft, the defect of the throwing plate is gradually compensated, and the aggregate efficiency is improved. When the rotating speed of the screw shaft is high, the force of the throwing plate on transferring particles exceeds the axial conveying capacity of the spiral blade.

The aggregate rate of the entire device shows a downward trend due to the lack of effective coordination between them. In summary, the aggregate efficiency of the spiral aggregate device first increases and then decreases with the increase in the rotating speed of the screw shaft, and the effect is the best when the rotating speed is $230 \mathrm{rpm}$ in the study. 


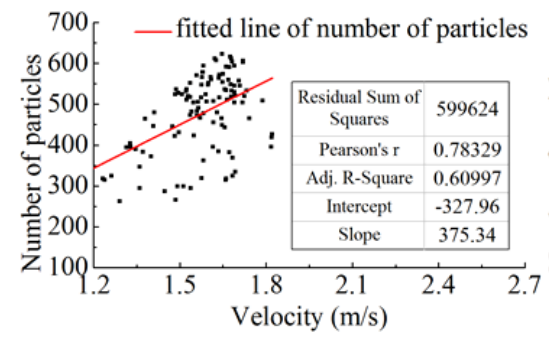

a) Rotation speed $166 \mathrm{rpm}$

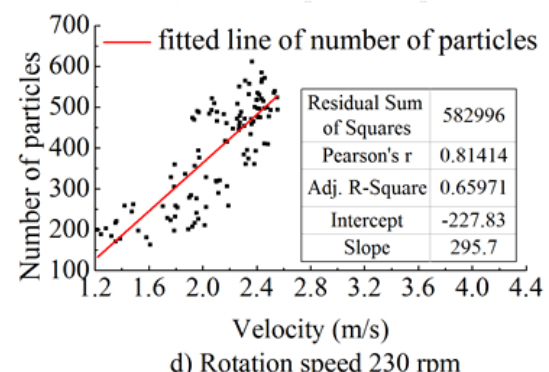

d) Rotation speed $230 \mathrm{rpm}$

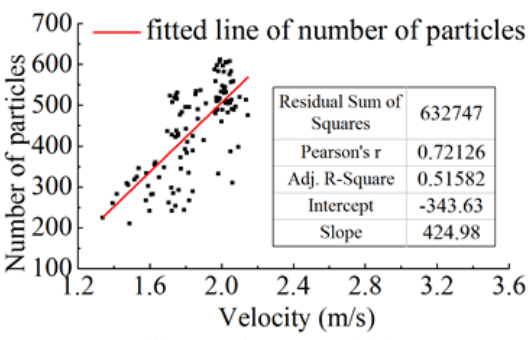

b) Rotation speed $200 \mathrm{rpm}$

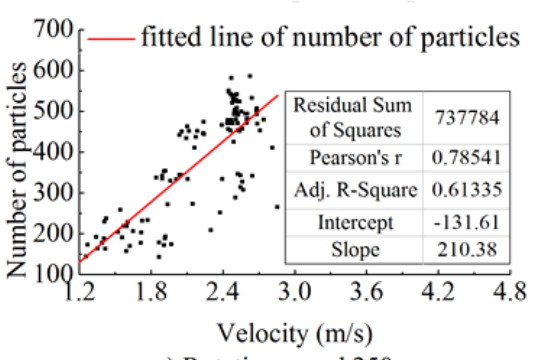

e) Rotation speed $250 \mathrm{rpm}$

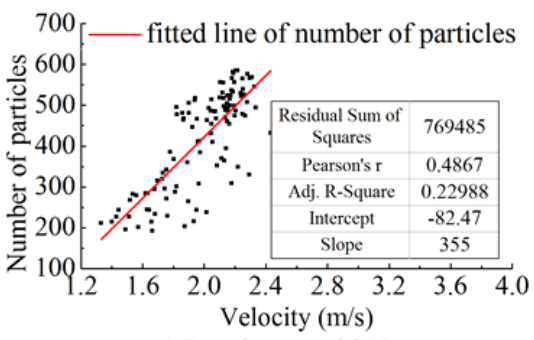

c) Rotation speed $215 \mathrm{rpm}$

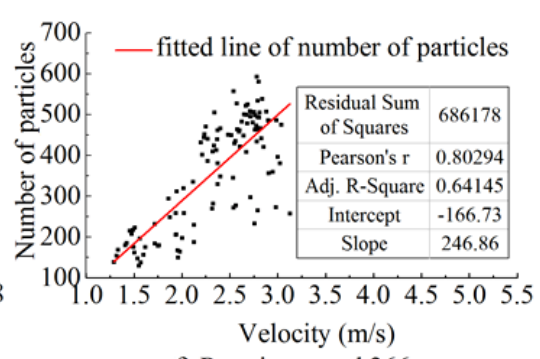

f) Rotation speed $266 \mathrm{rpm}$

Figure 4: Number of particles-velocity scatter plot and fitted line in different rotation speed.

\subsection{Analysis on the working process of the throwing plate}

Fig. 5 presents the simulation animation when the rotating speed of the screw shaft is $200 \mathrm{rpm}$, the slope of the roof of the feeding port is $13^{\circ}$, and the number of the throwing plates is 3 . According to the figure, the phenomenon of uneven working occurs among these throwing plates during work. The number of particles lifted by each plate when arriving at the feeding port is relatively fixed at each time, but the numbers of particles conveyed by the three throwing plates in a working cycle are obviously different from each other. The variation law of the number of particles in Area I must be analysed based on the spatial position of the throwing plate to determine the effect of the throwing plate on the periodic fluctuation of the number of particles in Area I.

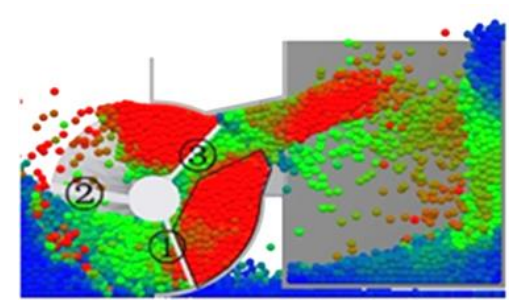

a) $2.5 \mathrm{~s}$

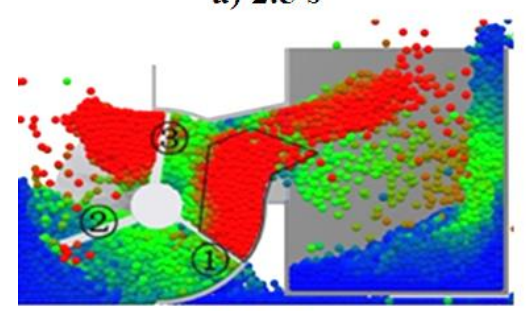

d) $2.78 \mathrm{~s}$

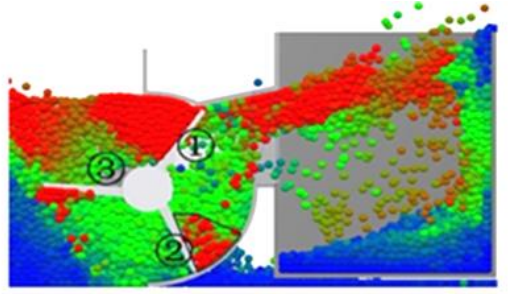

b) $2.59 \mathrm{~s}$

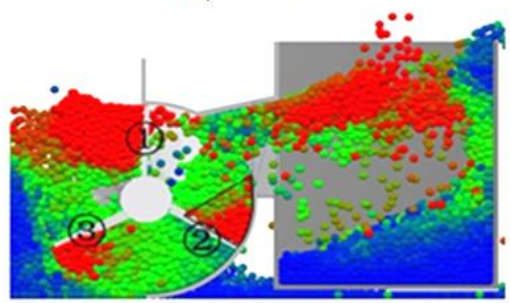

e) $2.87 \mathrm{~s}$

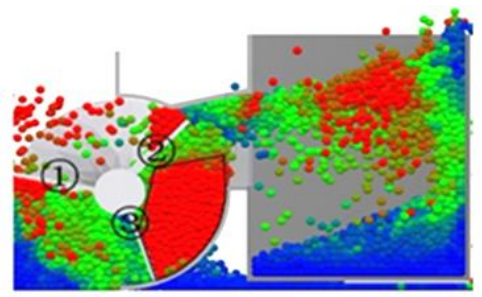

c) $2.67 \mathrm{~s}$

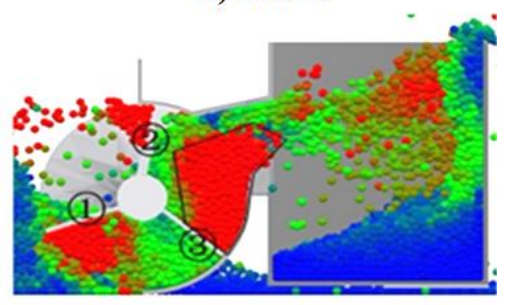

f) $2.95 \mathrm{~s}$

Figure 5: Comparison of the number of particles conveyed by different throwing plates.

According to Fig. 6 a, the throwing plates are numbered as (1), (2), and (3). The circle formed by the terminal turning track of the throwing plates is marked with dotted lines, and several key points on the circle are marked. Along the circumferential direction, an arc from the horizontal point to $\mathrm{C}$ is the boundary line between the rotating area of the screw shaft and the channel of the feeding port in the view direction. In this view, the throwing plates rotate 
counter clockwise around $\mathrm{O}$ point in the centre of the screw shaft. Thus, the end of the throwing plate would move from the horizontal point to $\mathrm{C}$. After analysis, when a throwing plate pointed at $\mathrm{C}$, its subsequent motion would not have a direct impact on the number of particles in Area I, and the change in the number of particles in Area I would depend on the effect of the next throwing plate on particles. From the angle of every two adjacent throwing plates $\left(120^{\circ}\right)$, when a throwing plate pointed at $\mathrm{C}$, the next throwing plate would point at $\mathrm{A}$ $\left(\angle A O C=120^{\circ}\right)$. Therefore, the motion process of the throwing plate from $\mathrm{A}$ to $\mathrm{C}$ is regarded as its main work stroke. According to this definition, the rotating process of the screw shaft for one cycle is divided into the following parts: No. 3 throwing plate points at A; No. 3 throwing plate points at $\mathrm{C}$ (No. 2 throwing plate points at $\mathrm{A}$ at the same time); No. 2 throwing plate points at $\mathrm{C}$ (No. 1 throwing plate points at $\mathrm{A}$ at the same time); No. 1 throwing plate points at $\mathrm{C}$.

Fig. $6 \mathrm{~b}$ shows the variation curve of the number of particles in Area I during the first cycle (1.17-2.07 s) of rotation of the screw shaft at the rotating speed of $166 \mathrm{rpm}$. Key points are marked as shown in Fig. 6 b. In the figure, (3/A represents that No. 3 throwing plate points at A, and other marks are the same. The peak number of particles in the work stroke of each throwing plate shows that the peak numbers of particles appear in the work strokes of No. 3 and No. 1 throwing plates when one of them points at B (the position of B is shown in Fig. 5 b), and the peak number of particles when No. 3 throwing plate points at B is obviously higher than that when No. 1 throwing plate points at B. However, the number of particles in the work stroke of No. 2 throwing plate increases monotonously, and the peak point cannot be found, which is significantly different from the working condition of the other two throwing plates. The time when No. 2 throwing plate points at B is also marked in its work stroke and no special mathematical characteristics are found. The above analysis method is extended to the entire simulation process. Comprehensive comparison and analysis confirmed that the above phenomenon is universal.

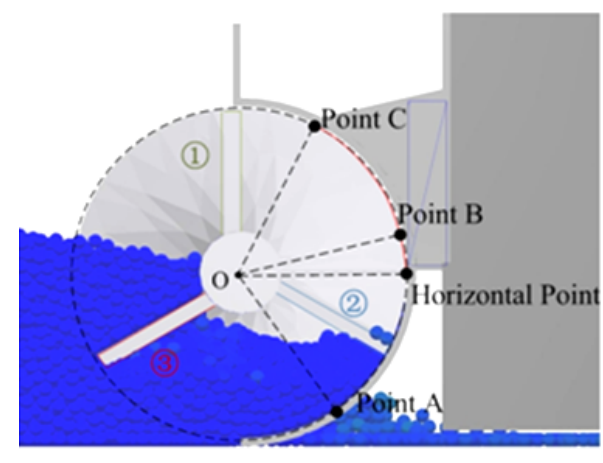

(a) The mark of the key points of the throwing plate

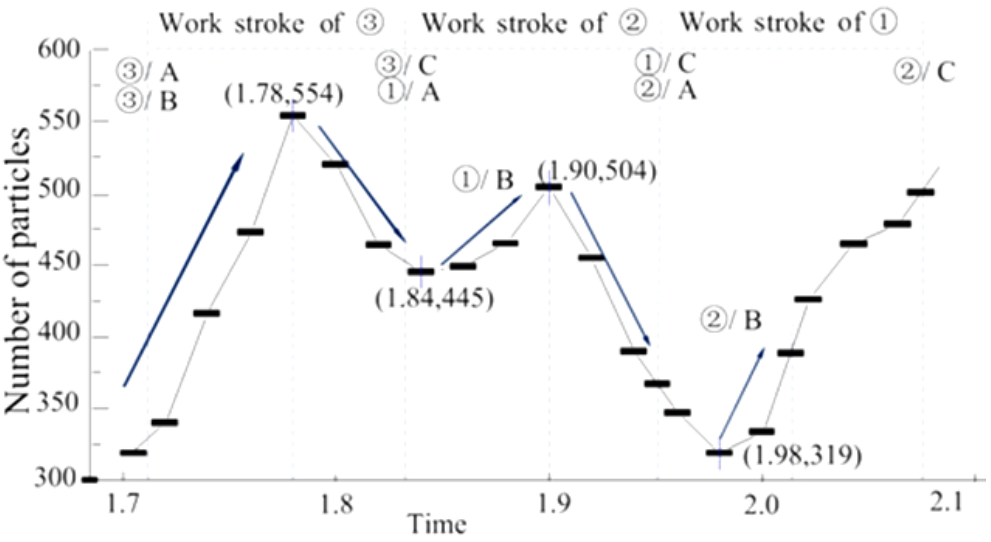

(b) The curve of the number of particles in Area I at $1.17 \mathrm{~s}-2.07 \mathrm{~s}$ with the screw shaft speed in $166 \mathrm{rpm}$.

Figure 6: Calibration and analysis of the spatial position of the throwing plates in a cycle.

\subsection{Influence of the number of throwing plates on the aggregate effect}

Fig. 7 shows the velocity variation curves of particles in Area II along the three directions of $\mathrm{x}, \mathrm{y}$, and $\mathrm{z}$ axes under different numbers of blades when the rotating speed of the screw shaft is $230 \mathrm{rpm}$ and the slope of the feeding port is $13^{\circ}$. In Area II, the upper and lower limits of the average velocity curve of particles along y axis are very small, and the average velocity is distributed basically at $0 \mathrm{~m} / \mathrm{s}$, which is determined by the symmetrical design of the screw conveyor. The throwing device can provide the projectile kinetic energy for particles collected at the front of the feeding port to enter the trough. However, because of the continuous 
circular motion of the device and the consistent speed with the rotating speed of the screw shaft, it would throw a part of them to the opposite direction while throwing materials into the trough, resulting in the phenomenon of material splashing forward. The splashing phenomenon may cause the average velocity of some particles along $\mathrm{x}$ axis to be positive. However, the integral value of any velocity curve along $x$ axis in Fig. 7 is less than 0 during 1.5 and $4 \mathrm{~s}$, which indicates that the splashing phenomenon of the throwing device is insufficient to affect its normal operation in this case. A reasonable control of the rotating speed of the screw shaft can make the work of the spiral aggregate device be mainly used to convey the materials to the rear trough.

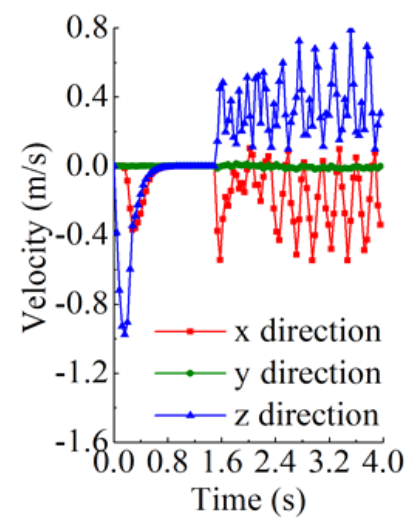

a) 2 throwing plates

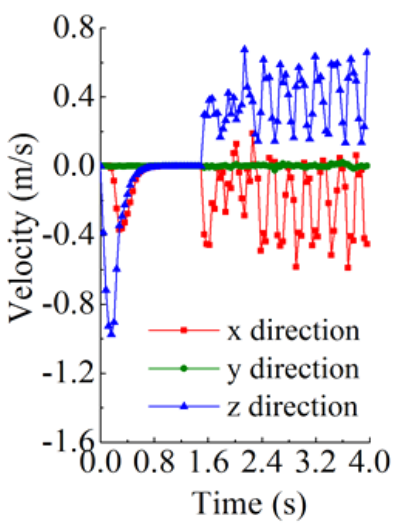

b) 3 throwing plates

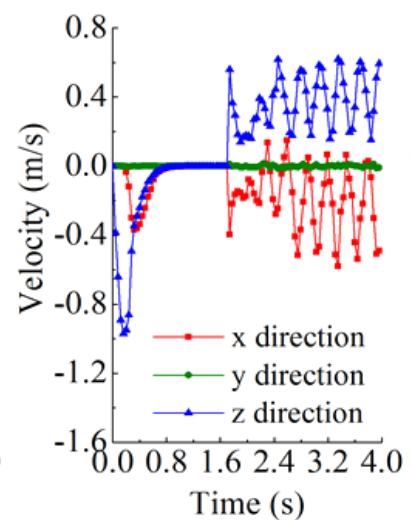

c) 4 throwing plates

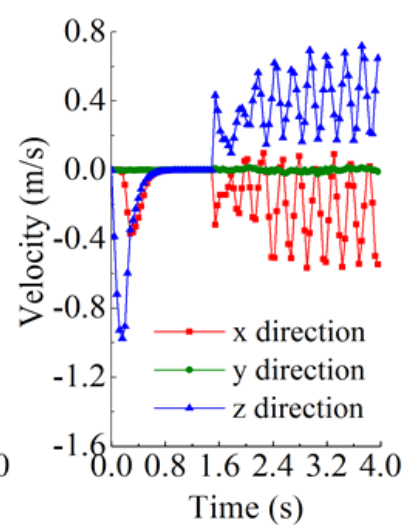

d) 5 throwing plates

Figure 7: Velocity variation curves of particles in Area II along the axes with different throwing plates.

Moreover, attention should be paid to the work matching between the spiral blade and throwing plate. Fig. 8 shows the variation in the average velocity of particles in Area III along $y$ axis with time under different numbers of throwing plates. From the figure, in the stable working stage after $2 \mathrm{~s}$, when the number of throwing plates is only 2, the average velocity of particles in y direction is small, and the velocity fluctuation is obvious, which indicates the lack of work of the throwing plate and the accumulation of particles in the middle. With the increase in the number of throwing plates, the average velocity is raised, and the velocity fluctuation also decreases. However, the volume of the throwing plate would also occupy the space in the middle of the spiral aggregate device. With the increase in the number of throwing plates, the occupied space gradually increases, which would hinder the movement of particles in the middle of the aggregate device. Thus, when the number of throwing plates is 5 , the velocity of particles in the y direction has a significant decline. Nevertheless, when the velocity fluctuation of particles is considered, a high number of throwing plates indicate that the work is performed numerous times on the particles in a rotating cycle. As a result, the velocity fluctuation of particles will be reduced.

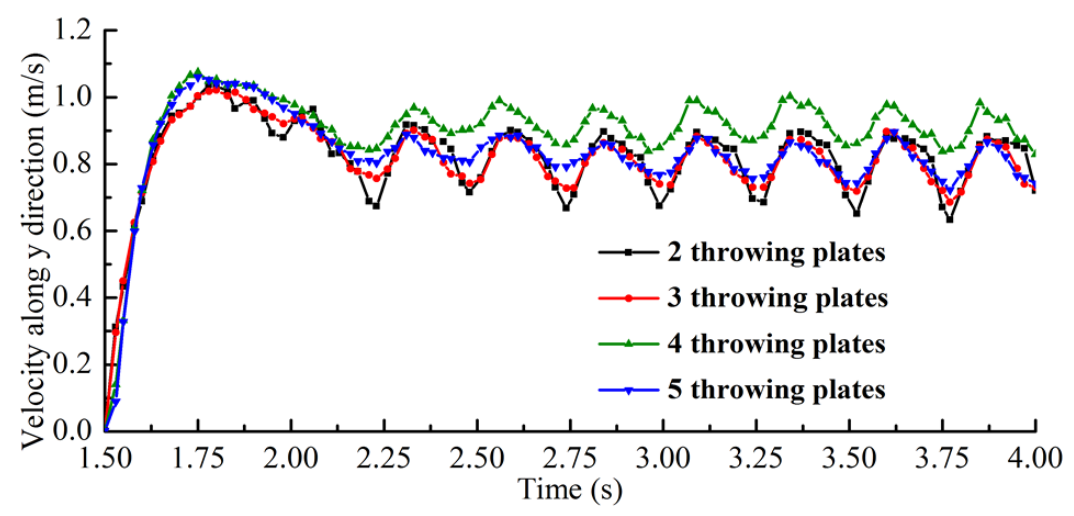

Figure 8: The variation of average velocity of particles in Area III along y axis. 
After comprehensive analysis, the work of the throwing plate with four blades could make the particles in the spiral blades obtain large axial speed after $2 \mathrm{~s}$ when the rotating speed of the screw shaft is $230 \mathrm{rpm}$. When the particles on both sides of the spiral blades are conveyed to the rotational space of the throwing plate in the middle, the throwing plate can feed these particles to the feeding port in time to reduce the accumulation of particles in the middle. Therefore, the four-blade throwing plate has high efficiency and a good working performance.

\subsection{Influence of the slope of feeding port on the aggregate effect}

Fig. 9 shows the velocity variation curves of particles in Area I with time along the three directions of $\mathrm{x}, \mathrm{y}$, and $\mathrm{z}$ axes under different inclination $\alpha$ when the rotating speed of the screw shaft is $230 \mathrm{rpm}$. Four curves in each figure (Fig. $9 \mathrm{a}, \mathrm{b}$, and c) coincide with one another and show similar trends, which indicates that the variation in the roof inclination on the feeding port has no significant effect on the velocity and direction of particle movement in the channel of the feeding port. To further analyse this problem, the animations of the four groups are compared. Fig. 10 presents the cross sections of the symmetrical centre of the simulation model at $1.96 \mathrm{~s}$ under four groups of simulation, in which the particles are represented by their velocity vector arrows; the colour of the arrows changed from blue to red, corresponding to the change in the movement velocity of particles from low to high. In the enlarged view of Fig. 10, two special areas are marked out, representing the accumulation triangle area and the empty quadrilateral area, which can be found in the cross sections of other simulation animations.

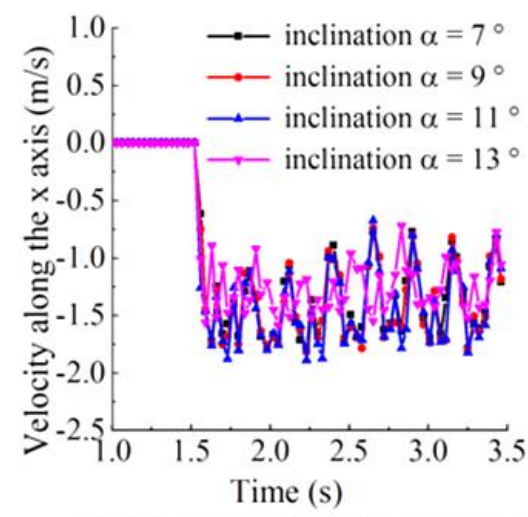

a) Particle velocity along the $x$ axis

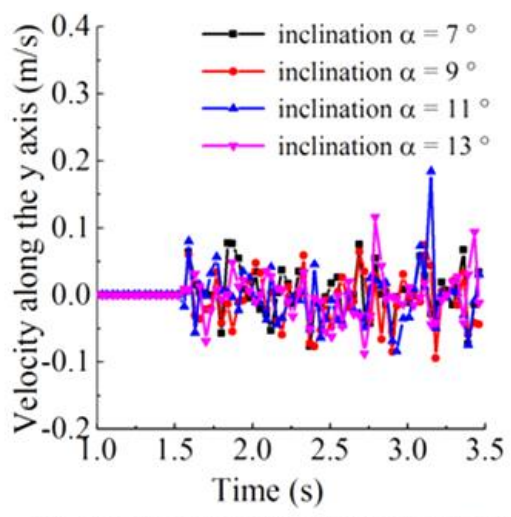

b) Particle velocity along the $y$ axis

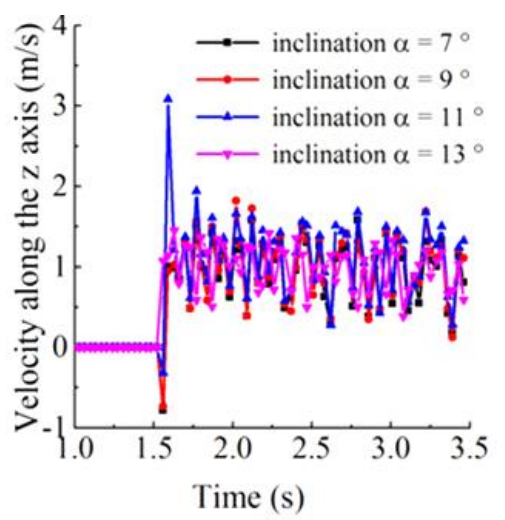

c) Particle velocity along the $z$ axis

Figure 9: The velocity of particles with time in Area I along each axis under different inclinations.

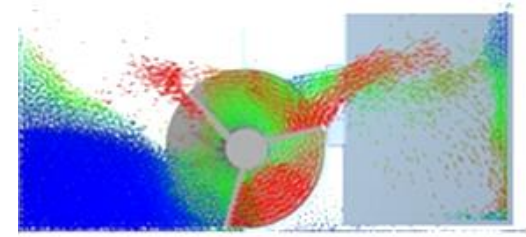

$\alpha=11^{\circ}$

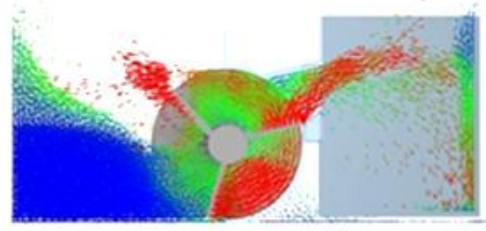

$\alpha=13^{\circ}$

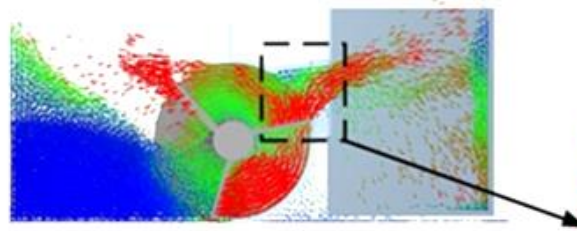

$\alpha=7^{\circ}$

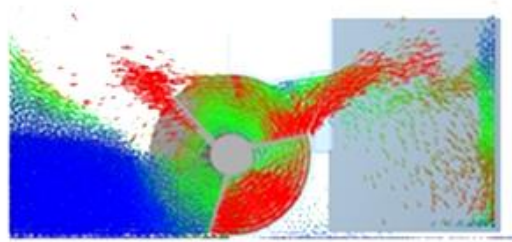

$\alpha=9^{\circ}$

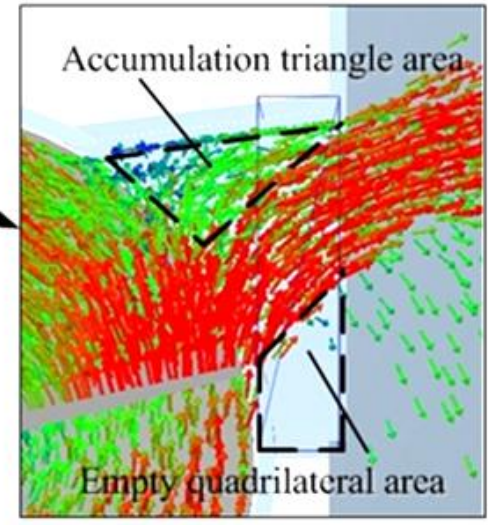

Enlarged view

Figure 10: Cross-section view of $1.96 \mathrm{~s}$ simulation model under different inclinations. 
The accumulation triangle area is located at the top of the feeding port channel. Its one side overlaps with the upper roof of the feeding port with particles of low velocity in it, and the area adjacent to the other two boundaries of the accumulation triangle area is filled with particles of high velocity. This finding indicates that during the working process of the spiral aggregate device, a part of particles accumulated at the top of the feeding port due to the continuous role of the throwing plate, occupying a relatively fixed triangle area. The accumulated particles here distribute the particles conveyed by the throwing plate into two parts, so that one part of the particles enters the trough through the feeding port, and the other part remains in the rotating space of the screw shaft and is finally thrown out due to the role of the throwing plate. The particles that passing through Area I do not directly contact the upper roof of the feeding port because of the accumulation of particles on the upper roof. Therefore, the slope change of the roof of the feeding port has no obvious effect on the conveying efficiency.

Another defect in the design of the mechanism is found from the current simulation results. The empty quadrilateral area marked in the magnified view of Fig. 10 is an area with very few particles in the channel of the feeding port, but it also appears in the simulations of different groups of parameters. Few particles pass through it, so there is no need to take this space as an open area for the channel of the feeding port. If the lower bottom of the channel of the feeding port is designed at the trapezoidal bevel edge of the empty quadrilateral area, it would not hinder the particles to enter the trough. Instead, it could increase the volume of the trough. Some space in the structure of the bottom floor is wasted, which needs further optimization.

\section{CONCLUSION}

To meet the design and optimization requirements of a spiral aggregate device, further improve the working efficiency of the sump cleaning machine, and accurately find the defects in previous design, the working process of a spiral aggregate device was simulated by means of DEM; the key components of the spiral device were optimized; and the influence of the rotating speed of the screw shaft, the number of throwing plates, and the slope of the feeding port on the aggregate effect was analysed. The following conclusions were reached.

(1) By using DEM, the working process of the key components of the spiral aggregate device could be effectively simulated, and the motion of particles at different positions, especially the internal state of particles that could not be observed by the naked eyes, could be given visually. The method could intuitively analyse the deficiencies in the structural design and thus realize the optimal design of the equipment.

(2) With increasing rotating speed of the screw shaft, the positive correlation between the speed and number of particles in the channel of the feeding port increases first and decreases later. The particle conveying efficiency also shows a trend of increasing first and decreasing later. With the increase of the number of throwing plates, the fluctuation of the axial flowing speed of particles along the screw shaft decreases gradually, but the speed first increases and then decreases. When the rotating speed of the screw shaft is $230 \mathrm{rpm}$, the four-blade throwing plate has a better working performance, and the aggregate efficiency is the highest.

(3) Particles would be accumulated on the upper roof of the feeding port. As a result, the change in slope has no obvious effect on the conveying efficiency, and the structure of the bottom plate of the feeding port channel hinders the entry of particles. To make the particles pass through the feeding port efficiently and ensure the depth of the aggregate trough, the arc of the bottom plate of the feeding port should be reduced, or other structural forms should be adopted.

In the study, DEM was applied to the simulation of the spiral conveyor. The simplified simulation model not only highlights the structural characteristics of the device but also 
improves the calculation efficiency, which provides a reference for the structural optimization and performance analysis of the spiral aggregate device and an important supplement to the performance research of the spiral conveying device with a special structure. Some defects of the feeding port structure on the spiral aggregate device are found, which need to be improved. Therefore, in future research, the influence of the structure of the feeding port would be studied by using DEM, the detailed optimization design and improvement scheme would be given, and the optimized prototype would be trial produced for industrial test.

\section{ACKNOWLEDGEMENT}

This work was supported by the National Natural Science Foundation of China (Grant No. 51704178), National Natural Science Foundation of Shandong Province (Grant No. ZR2017MEE034), China Postdoctoral Science Foundation funded project (Grant No. 2018T110700) and Foundation of Jiangsu Key Laboratory of Mine Mechanical and Electrical Equipment (Grant No. JSKL-MMEE-2018-1).

\section{REFERENCES}

[1] Li, B.; Wei, J. P.; Sun, D. H.; Zhang, L. L.; Borisyukd, Y. A. (2016). Research on the reasonable spacing of holes in gas drainage along coal seams in consideration of the superimposed effect of drainage, Journal of Engineering Science and Technology Review, Vol. 9, No. 1, 102-110, doi:10.25103/jestr.091.16

[2] Qiu, J. W.; Liu, Z. G.; Zhou, L.; Qin, R. X. (2017). Prediction model of gas quantity emitted from coal face based on PCA-GA-BP neural network and its application, Journal of Power Technologies, Vol. 97, No. 3, 169-178

[3] Li, H.; Bai, H. B.; Wu, J. J.; Zhao, H. M.; Ma, K. (2017). A method for prevent water inrush from karst collapse column: a case study from Sima mine, China, Environmental Earth Sciences, Vol. 76, No. 14, Paper 493, doi:10.1007/s12665-017-6831-2

[4] Hu, Z.-W.; Du, C.-L.; Hu, B. (2011). Optimum design of screw mixer of new sump cleaning device based on ant colony algorithm, Coal Mine Machinery, Vol. 32, No. 4, 13-15, doi:10.3969/j.issn.1003-0794.2011.04.006

[5] Feng, L.; Liu, J.-T.; Zhang, M.-Q.; Song, L.-L. (2010). Analysis on influencing factors of sedimentation characteristics of coal slime water, Journal of China University of Mining and Technology, Vol. 39, No. 5, 671-675

[6] Deng, X. L.; Zheng, K.; Davé, R. N. (2018). Discrete element method based analysis of mixing and collision dynamics in adhesive mixing process, Chemical Engineering Science, Vol. 190, 220-231, doi:10.1016/j.ces.2018.06.043

[7] Hang, C. G.; Huang, Y. X.; Zhu, R. X. (2017). Analysis of the movement behaviour of soil between subsoilers based on the discrete element method, Journal of Terramechanics, Vol. 74, 35-43, doi:10.1016/j.jterra.2017.10.002

[8] Kuruneru, S. T. W.; Sauret, E.; Saha, S. C.; Gu, Y. T. (2017). A coupled finite volume \& discrete element method to examine particulate foulant transport in metal foam heat exchangers, International Journal of Heat and Mass Transfer, Vol. 115, Part A, 43-61, doi:10.1016/j.ijheatmasstransfer.2017.07.027

[9] Wang, X. W.; Li, B.; Wang, S. W.; Yang, Z. J.; Cai, L. (2017). The transporting efficiency and mechanical behavior analysis of scraper conveyor, Proceedings of the Institution of Mechanical Engineers, Part C: Journal of Mechanical Engineering Science, Vol. 232, No. 18, 3315-3324, doi: $10.1177 / 0954406217734002$

[10] Ilic, D.; Wheeler, C. A. (2017). Transverse bulk solid behaviour during discharge from troughed belt conveyors, Advanced Powder Technology, Vol. 28, No. 9, 2410-2430, doi:10.1016/ j.apt.2017.06.024

[11] Sinnott, M. D.; Cleary, P. W. (2017). Particulate and water mixing in the feed box for a screen, Minerals Engineering, Vol. 109, 109-125, doi:10.1016/j.mineng.2017.03.008 
[12] Gao, K. D.; Du, C. L.; Dong, J. H.; Zeng, Q. L. (2015). Influence of the drum position parameters and the ranging arm thickness on the coal loading performance, Minerals, Vol. 5, No. 4, 723-736, doi: $10.3390 / \min 5040520$

[13] Roberts, A. W. (1999). The influence of granular vortex motion on the volumetric performance of enclosed screw conveyors, Powder Technology, Vol. 104, No. 1, 56-67, doi:10.1016/S00325910(99)00039-X

[14] Dai, J.; Grace, J. R. (2008). A model for biomass screw feeding, Powder Technology, Vol. 186, No. 1, 40-55, doi:10.1016/j.powtec.2007.10.032

[15] Lessmann, J.-S.; Schoeppner, V. (2015). Validation of discrete element simulations in the field of solids conveying in single-screw extruders, AIP Conference Proceedings (The $30^{\text {th }}$ International Conference of the Polymer Processing Society), Vol. 1664, No. 1, Paper 050001, 5 pages, doi:10.1063/1.4918405

[16] Nachenius, R. W.; van de Wardt, T. A.; Ronsse, F.; Prins, W. (2015). Residence time distributions of coarse biomass particles in a screw conveyor reactor, Fuel Processing Technology, Vol. 130, 87-95, doi:10.1016/j.fuproc.2014.09.039

[17] Davison, J.; Calay, R.; Sands, T.; England, M. (2002). CFD simulation of soil flow over augers, ASME 2002 Pressure Vessels and Piping Conference, Vol. 1, 197-203, doi:10.1115/PVP2002$\underline{1480}$

[18] Davison, J. B.; Calay, R. K.; Sands, T. B. (2005). Computational simulation of rheologically classified soil flow over augers, Journal of Marine Engineering \& Technology, Vol. 4, No. 1, 23 31, doi:10.1080/20464177.2005.11020185

[19] Talebi, K.; Memarian, H.; Rostami, J.; Gharahbagh, E. A. (2015). Modeling of soil movement in the screw conveyor of the earth pressure balance machines (EPBM) using computational fluid dynamics, Tunnelling and Underground Space Technology, Vol. 47, 136-142, doi:10.1016/ j.tust.2014.12.008

[20] Jovanović, A.; Pezo, L.; Stanojlović, S.; Kosanić, N.; Lević, L. (2015). Discrete element modelling of screw conveyor-mixers, Hemijska industrija, Vol. 69, No. 1, 95-101, doi:10.2298/HEMIND130412026J

[21] Ayllon-Perez, J.; Coello-Sobrino, J.; Martinez-Martinez, A.; Medina-Rios, N.; Miguel-Eguia, V. (2016). Evaluation of behavior under tensile loads of composite materials hybrid woven vinylester matrix by the finite element method, DYNA, Vol. 91, No. 6, 647-653, doi: $10.6036 / 7946$

[22] Patwa, A.; Ambrose, R. P. K.; Dogan, H.; Casada, M. (2014). Wheat mill stream properties for discrete element method modeling, Transactions of the ASABE, Vol. 57, No. 3, 891-899, doi: $10.13031 / \operatorname{trans} .57 .10626$

[23] Tsuji, Y.; Tanaka, T.; Ishida, T. (1992). Lagrangian numerical simulation of plug flow of cohesionless particles in a horizontal pipe, Powder Technology, Vol. 71, No. 3, 239-250, doi:10.1016/0032-5910(92)88030-L

[24] Cundall, P. A.; Strack, O. D. L. (1979). A discrete numerical model for granular assemblies, Geotechnique, Vol. 29, No. 1, 47-65, doi:10.1680/geot.1979.29.1.47 